\title{
BMJ Open Effectiveness and feasibility of telerehabilitation in patients with COVID-19: a protocol for a systematic review and meta-analysis
}

\author{
Abubeker Alebachew Seid (D) , ${ }^{1}$ Setognal Birara Aychiluhm (D) , \\ Ahmed Adem Mohammed ${ }^{1}$
}

\section{To cite: Seid AA,}

Aychiluhm SB, Mohammed AA. Effectiveness and feasibility of telerehabilitation in patients with COVID-19: a protocol for a systematic review and meta-analysis. BMJ Open 2022;12:e058932. doi:10.1136/ bmjopen-2021-058932

- Prepublication history for this paper is available online. To view these files, please visit the journal online (http://dx.doi org/10.1136/bmjopen-2021058932).

Received 03 November 2021 Accepted 15 December 2021

D Check for updates

(C) Author(s) (or their employer(s)) 2022. Re-use permitted under CC BY-NC. No commercial re-use. See rights and permissions. Published by BMJ.

${ }^{1}$ Department of Nursing, College of Medicine and Health Sciences, Samara University, Samara, Ethiopia

${ }^{2}$ Department of Public Health, College of Medicine and Health Sciences, Samara University, Samara, Ethiopia

Correspondence to Abubeker Alebachew Seid; abubeker2008h@gmail.com

\section{ABSTRACT}

Introduction Respiratory rehabilitation is the use of exercise, education, and behavioural interventions to alleviate symptoms and improve quality of life. Recent studies highlight that respiratory rehabilitation is effective and safe for patients with COVID-19. We aim to evaluate the effectiveness and feasibility of respiratory telerehabilitation on patients infected with COVID-19 by conducting a systematic review and meta-analysis. Methods and analysis PubMed, Web of Science, Science Direct, Physiotherapy Evidence Database, Google Scholar and Cochrane Library databases will be searched from inception to the end of November 2021. Randomised controlled trials investigating the effectiveness of telerehabilitation in the management of COVID-19 will be included. The primary outcomes will be functional capacity, cardiopulmonary exercise tests and quality of life. Secondary outcomes will include anxiety/depression level, sleep quality, mortality rate, completion rate, reason for withdrawal, adverse events, service satisfaction, costeffectiveness and other potential factors. Two reviewers will independently screen and extract data and perform quality assessment of included studies. The Cochrane risk of bias tool will be used to assess risk of bias. Review Manager V.5.4 (Cochrane Collaboration) software will be used for statistical analysis. Heterogeneity will be analysed using $\mathrm{l}^{2}$ statistics. Mean difference or standardised mean difference with $95 \% \mathrm{Cl}$ and $\mathrm{p}$ value will be used to calculate treatment effect for outcome variables.

Ethics and dissemination Ethical approval is not required because this systematic review and metaanalysis is based on previously published data. Final result will be published in peer-reviewed journal and presented at relevant conferences and events.

PROSPERO registration number CRD42021287975.

\section{INTRODUCTION}

COVID-19 is a highly infectious respiratory disease, which leads to respiratory, physical and psychological dysfunctions. ${ }^{12}$ COVID-19 has clinical manifestations of cough, shortness of breath, chest pain and so on. Respiratory rehabilitation improves symptoms of dyspnoea, relieves anxiety, reduces
Strengths and limitations of this study

Data screening, extraction and methodological quality will be performed by two reviewers independently.

- This study will be reported according to Preferred Reporting Items for Systematic Reviews and MetaAnalyses guidelines.

- Standardised methodological appraisal tools will be used to assess risk of bias of the included studies in the review.

- Egger's and Begg's tests will be conducted for the assessment of the publication bias.

- The absence of sufficient high-quality randomised controlled trials, heterogeneity in the interventions, high dropout and small sample size might be the limitations for this review.

complications, minimises disability, preserves function and improves quality of life both in the acute phase and after discharge. ${ }^{23}$ Some of the respiratory physiotherapy interventions include airway clearance techniques, non-invasive ventilation and inspiratory positive pressure breathing, secretion clearance techniques, exercise and mobilisation, and many other techniques. ${ }^{2-4}$

Telerehabilitation is the provision of rehabilitation services through telecommunication networks or the internet offering remote treatments to the people in their homes or from a distance. ${ }^{56}$ Since COVID-19 emerged and caused the collapse of health systems, many patients are not able to receive their face-to-face treatments. Chronic patients are unable to continue their follow-up as usual, professionals could not attend all of the consultations and the high contagious nature of the disease forced a new treatment approach, that is, telerehabilitation to be used widely. ${ }^{6}$

Telerehabilitation had found to improve exercise capacity, self-efficacy and mood in 
patients with chronic obstructive pulmonary disease (COPD). Telerehabilitation also provided good patient adherence and less dropout throughout the intervention, which indicated that supervision of in-home exercise training using video conferencing is feasible and has benefits for patients with COPD. ${ }^{8-10}$ Telemedicine has great potential for connecting patients and healthcare professionals, while respecting social safety restrictions. Digital health interventions can help provide self-monitoring tools, field updates, exercise protocols and psychological support. ${ }^{4}$

Telerehabilitation can be provided with applications via chat or video calling (eg, RespiraConNosotros, RehabApp), virtual reality, live talks, telephone, internet with or without supervision and at hospitals or health centres. ${ }^{611-14}$ Scientific literature has explored the effectiveness of these treatments in different chronic pathologies such as diabetes mellitus, chronic lung disease, cardiovascular disease, and respiratory conditions, such as COPD or cystic fibrosis. ${ }^{6}$

It was mentioned in many research papers that respiratory telerehabilitation plays an important role in the recovery of patients from COVID-19. The first ever randomised controlled trial (RCT) aimed to evaluate exercise capacity, lower limb muscle strength (LMS), pulmonary function, health-related quality of life (HRQOL) and dyspnoea found that telerehabilitation improves functional exercise capacity, LMS and physical HRQOL but no improvements in pulmonary function tests and mental aspect of quality of life. ${ }^{3}$ A recent systematic review on rehabilitation of patients in post-COVID-19 infection suggested that respiratory rehabilitation interventions improve pulmonary function, physical and psychological efficiency, and quality of life. But this study had limitations due to a lack of RCTs included in the review. ${ }^{4}$

It is unclear whether respiratory telerehabilitation could improve outcomes in patients with COVID-19 due to limited original research. To the best of our knowledge, this is the first review to investigate the effectiveness and feasibility of telerehabilitation in patients with COVID-19 in the literature. Thus, the aims of this systematic review and meta-analysis are twofold. First, we will evaluate the effectiveness of respiratory telerehabilitation in patients with COVID-19. Second, we will discuss the feasibility of respiratory telerehabilitation and potential contributing factors, and we will investigate and summarise completion rates, reasons for withdrawal, service satisfaction and cost-effectiveness of the interventions.

\section{METHODS AND ANALYSIS}

\section{Search strategy}

Literature search will be carried out in six databases: PubMed, Web of Science, Science Direct, Physiotherapy Evidence Database, Google Scholar, and Cochrane Library databases, and articles published from inception to the end of November 2021 will be included. The following search terms will be used: "COVID-19"

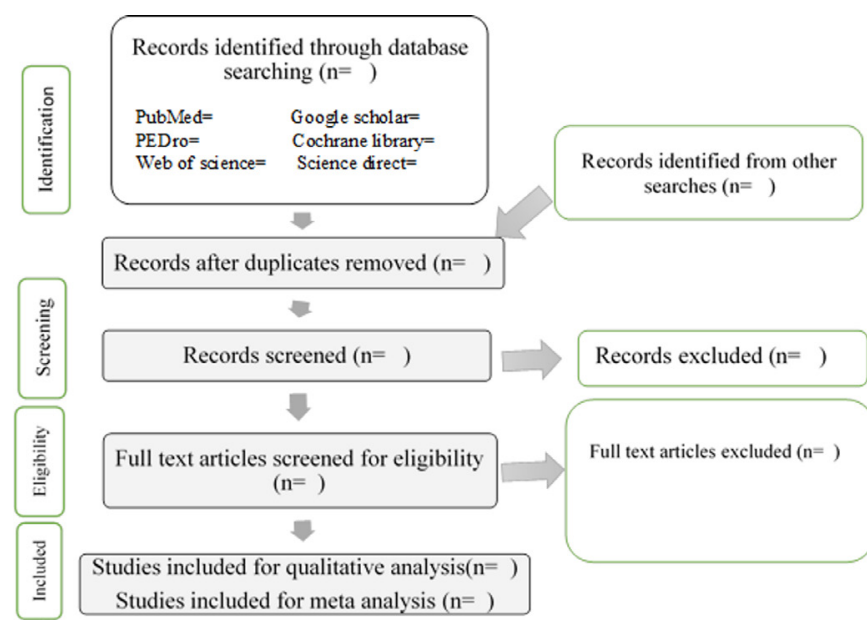

Figure 1 Preferred Reporting Items for Systematic Reviews and Meta-analyses flow chart describing the search strategy.

OR “COVID-19” OR "novel coronavirus 2019” OR "novel coronavirus disease 2019" OR "2019-nCoV" OR "SARS-CoV" OR "SARS-CoV-2" OR "corona virus 2019" OR "new corona virus" OR "COVID-19 19 disease" AND "tele-rehabilitation" OR "telerehabilitation" OR "respiratory rehabilitation" OR "respiratory physiotherapy" OR "pulmonary rehabilitation" OR "pulmonary physiotherapy" AND "randomized controlled trial". The literature selection process will be conducted according to the Preferred Reporting Items for Systematic Reviews and Meta-analyses guidelines ${ }^{15}$ and presented in figure 1 . RCTs concerning the effects of telerehabilitation programmes for patients with COVID-19 will be included in this systematic review and meta-analysis. Two reviewers will independently select studies, and any disagreement between the reviewers will be resolved by consensus or by another third reviewer. The detailed search strategy of PubMed is presented in table 1 .

\section{Inclusion criteria}

RCTs comparing telerehabilitation with any rehabilitation programme in patients with COVID-19 in the acute or long-term follow-up will be included in this review. Telerehabilitation is defined as any rehabilitation programme delivered by physiotherapy professionals via telecom/internet network services to patients with COVID-19. Telerehabilitation for COVID-19 might include aerobic training (such as walking, fast walking, jogging, swimming, etc), progressive strength training, secretion drainage or ventilatory techniques, aerobic, flexibility and strengthening exercises for upper and lower extremity, breathing/respiratory exercise and other physical training programmes. ${ }^{11} 1216$ Two reviewers will independently assess the titles and abstracts, and fulltext published RCTs in English language will be included.

\section{Outcome measures}

The primary outcomes of interest will be functional capacity (eg, 6 min walking distance), cardiopulmonary exercise tests and quality of life. Secondary outcomes 
Table 1 PubMed search strategy

\begin{tabular}{|c|c|}
\hline Search number & Search detail \\
\hline$\# 1$ & “COVID-19”[MeSH Terms] \\
\hline \#2 & $\begin{array}{l}\text { "COVID-19"[Title/Abstract]OR "COVID-19"[Title/Abstract]OR "novel coronavirus 2019" [Title/Abstract]OR } \\
\text { "novel coronavirus disease 2019"[Title/Abstract]OR "2019-nCoV”[Title/Abstract]OR "SARS-CoV"[Title/ } \\
\text { Abstract]OR "SARS-CoV-2"[Title/Abstract]OR "corona virus 2019"[Title/Abstract]OR "new corona } \\
\text { virus"[Title/Abstract]OR "COVID-19 } 19 \text { disease"[Title/Abstract]OR "tele-rehabilitation"[Title/Abstract]OR } \\
\text { "telerehabilitation"[Title/Abstract] }\end{array}$ \\
\hline \#5 & \#3 AND \#4 \\
\hline
\end{tabular}

of interest will be anxiety and depression scales, sleep quality, mortality rate and smoking cessation. Feasibility outcomes of interest will include intervention completion rate, reason for withdrawal, adverse events, service satisfaction and cost-effectiveness. Other potential contributing factors for feasibility like information communication technology skill and experience, age and medical condition will be analysed where data are available.

\section{Data extraction}

Reviewers will independently extract the data on a standard worksheet and disagreements will be resolved by consensus or with the help of a third reviewer. The following information will be extracted from each included trial: author, year of publication, country of publication, inclusion/exclusion criteria, sample characteristics, number of participants, experimental and control interventions detail, duration of intervention, follow-up, outcomes and results. For missing data, we will contact the corresponding authors of the studies through email.

\section{Methodological quality assessment}

The risk of bias of the included trials will be assessed using the Cochrane risk of bias tool for RCTs. ${ }^{17}$ The reviewers will reach concurrence on the final score of all the included trials and the result will be displayed in a table or graph. Grading of Recommendations, Assessment, Development and Evaluation will be used for assessment of the quality of evidence for outcomes and results will be categorised as 'high', 'moderate', 'low' or 'very low' accordingly. ${ }^{18}$ Two authors (AAS and SAB) will rate independently and a third author (AAM) will address any discrepancy. Publication bias across studies will be examined using funnel plot method, Egger's test and Begg's test ${ }^{19}$ using the Stata V.16.0 software.

\section{Data synthesis}

Review Manager V.5.4 (Cochrane Collaboration) software will be used to conduct the meta-analysis. The mean difference or standardised mean difference will be used to analyse continuous variables with $95 \% \mathrm{CI}$ with corresponding $\mathrm{p}$ value. Heterogeneity among included trials will be assessed using the $\mathrm{I}^{2}$ test. First, a fixed-effects model will be used for data analysis. If $\mathrm{I}^{2}>0.5$ or $\mathrm{p}<0.1$, it is considered that there is a significant heterogeneity among the included trials, ${ }^{2}$ and random-effects model will be used in this case. To determine the source of heterogeneity, sensitivity analysis will be conducted by excluding trials one by one.

\section{Patient and public involvement \\ No patient involved.}

Contributors All authors have made significant contributions to this study protocol. AAS developed the research question, wrote the first draft, designed the search strategy, and edited and approved the final version of the manuscript. SAB developed the research question, revised the search strategy of databases, developed the data extraction form, and edited and approved the final version of the manuscript. AAM revised the data extraction form and edited and approved the final version of the manuscript.

Funding The authors have not declared a specific grant for this research from any funding agency in the public, commercial or not-for-profit sectors.

Competing interests None declared.

Patient and public involvement Patients and/or the public were not involved in the design, or conduct, or reporting, or dissemination plans of this research.

Patient consent for publication Not required.

Provenance and peer review Not commissioned; externally peer reviewed.

Open access This is an open access article distributed in accordance with the Creative Commons Attribution Non Commercial (CC BY-NC 4.0) license, which permits others to distribute, remix, adapt, build upon this work non-commercially, and license their derivative works on different terms, provided the original work is properly cited, appropriate credit is given, any changes made indicated, and the use is non-commercial. See: http://creativecommons.org/licenses/by-nc/4.0/. 


\section{ORCID iDs}

Abubeker Alebachew Seid http://orcid.org/0000-0002-9171-3211

Setognal Birara Aychiluhm http://orcid.org/0000-0001-7565-7515

\section{REFERENCES}

1 Thomas $\mathrm{P}$, Baldwin C, Bissett B, et al. Physiotherapy management for COVID-19 in the acute hospital setting: clinical practice recommendations. J Physiother 2020;66:73-82.

2 Zhu F, Zhang M, Gao M, et al. Effects of respiratory rehabilitation on patients with novel coronavirus (COVID-19) pneumonia in the rehabilitation phase: protocol for a systematic review and metaanalysis. BMJ Open 2020;10:e039771.

3 Li Jian'an, Xia W, Zhan C. Effectiveness of a telerehabilitation program for COVID-19 survivors (TERECO) on exercise capacity, pulmonary function, lower limb muscle strength, and quality of life: a randomized controlled trial. medRxiv 2021.

4 Demeco A, Marotta N, Barletta M, et al. Rehabilitation of patients post-COVID-19 infection: a literature review. J Int Med Res 2020;48:030006052094838.

5 Bairapareddy KC, Chandrasekaran B, Agarwal U. Telerehabilitation for chronic obstructive pulmonary disease patients: an underrecognized management in tertiary care. Indian J Palliat Care 2018;24:529-33.

6 Bermejo-Gil BM, Pérez-Robledo F, Llamas-Ramos R. Valderi Reis Quietinho Leithardt IL-R. RespiraConNosotros: a viable home-based telerehabilitation system for respiratory patients. Sensors 2021;21.

7 Adly AS, Adly MS, Adly AS. Telemanagement of Home-Isolated COVID-19 patients using oxygen therapy with noninvasive positive pressure ventilation and physical therapy techniques: randomized clinical trial. J Med Internet Res 2021;23:e23446.

8 Holland AE, Cox NS. Telerehabilitation for COPD: could pulmonary rehabilitation deliver on its promise? Respirology 2017;22:626-7.

9 Tsutsui M, Gerayeli F, Sin DD. Pulmonary rehabilitation in a PostCOVID-19 world: Telerehabilitation as a new standard in patients with COPD. Int J Chron Obstruct Pulmon Dis 2021;16:379-91.
10 Vasilopoulou M, Papaioannou Al, Kaltsakas G, et al. Homebased maintenance tele-rehabilitation reduces the risk for acute exacerbations of COPD, hospitalisations and emergency department visits. Eur Respir J 2017;49. doi:10.1183/13993003.02129-2016. [Epub ahead of print: 2505 2017].

11 Turan Z, Mahir Topaloglu OOT. Is tele-rehabilitation superior to home exercise program in COVID-19 survivors following discharge from intensive care unit? - a study protocol of a randomized controlled trial. Physiother Res Int 1920.

12 Pastora-Bernal J-M, Estebanez-Pérez M-J, Molina-Torres G, et al. Telerehabilitation intervention in patients with covid-19 after hospital discharge to improve functional capacity and quality of life. study protocol for a multicenter randomized clinical trial. Int J Environ Res Public Health 2021;18. doi:10.3390/ijerph18062924. [Epub ahead of print: 1203 2021].

13 CB-UJJG-GMS-H EA-L, Manuel Perez-Ale CR-B. Short-term effects of a respiratory telerehabilitation program in confined covid-19 patients in the acute phase: a pilot study. Int $J$ Environ Res Public Heal:7511.

14 da Silva TD, de Oliveira PM, Dionizio JB, et al. Comparison between conventional intervention and Non-immersive virtual reality in the rehabilitation of individuals in an inpatient unit for the treatment of COVID-19: a study protocol for a randomized controlled crossover trial. Front Psychol 2021;12:622618.

15 Moher D, Shamseer L, Clarke M, et al. Preferred reporting items for systematic review and meta-analysis protocols (PRISMA-P) 2015 statement. Syst Rev 2015;4:1.

16 Gonzalez-Gerez JJ, Bernal-Utrera C, Anarte-Lazo E, et al. Therapeutic pulmonary telerehabilitation protocol for patients affected by COVID-19, confined to their homes: study protocol for a randomized controlled trial. Trials 2020;21.

17 Higgins JPT, Altman DG, Gøtzsche PC, et al. The Cochrane collaboration's tool for assessing risk of bias in randomised trials. BMJ 2011;343:d5928.

18 Guyatt GH, Oxman AD, Vist GE, et al. Grade: an emerging consensus on rating quality of evidence and strength of recommendations. $B M J$ 2008;336:924-6.

19 Vandenbroucke JP. Bias in meta-analysis detected by a simple, graphical test. experts' views are still needed. BMJ 1998;316:469-70. 